\title{
DETECÇÃo DE OSCILAÇÃo E CAUSA RAIZ EM UMA PLANTA TERMOELÉTRICA
}

\author{
CARlos Eduardo C. DE MELO ${ }^{1}$, CELSO J. MUNARO ${ }^{2}$
}

1. Departamento de Engenharia Elétrica e Eletrônica, ArcelorMittal Tubarão-ES

Rua Brigadeiro Eduardo Gomes, 930, Jardim Limoeiro, Vitória, ES, 29163-970

E-mails: carlos.eduardomelodarcelormittal.com.br

\author{
2. Universidade Federal do Espírito Santo, Depto. de Engenharia Elétrica \\ Av. Fernando Ferrari, 514, Goiabeiras, Vitória, ES, 29075-910 \\ E-mails: cjmunaro@gmail.com
}

\begin{abstract}
This article addresses the problem of detection of oscillations and root cause analysis for a thermoelectric power plant. The well-known method to detect oscillations based on autocorrelation function is used, which detects the signals with similar frequencies of oscillation and energy greater than a given threshold in this frequency band. Considering that these clustered signals are somehow related, an algorithm based on non-parametric identification has been proposed to indicate the root cause of this oscillation by finding the direction of the dependence between the signals. This approach has the advantage of not requiring the model order, dead time estimation and the definition of a model structure. The method is illustrated using a simulation example and also data collected from an industrial plant, with results validated using the process knowledge.
\end{abstract}

Keywords — Identificação de sistemas, Diagnóstico de falhas, Detecção de Oscilações, Dynamic Modelling, Time Series.

Resumo - Este artigo trata o problema de detecção de oscilações e análise da causa raiz de uma planta termoelétrica industrial. Para detectar as oscilações é usado o método já conhecido baseado na função de auto-correlação, que detecta os sinais com frequências de oscilação semelhantes, e que contenham energia maior que um certo limiar nesta banda de frequências. Considerando que estes grupos de sinais estão relacionados de alguma forma, um algoritmo baseado em identificação não paramétrica é proposto para indicar a causa raiz desta oscilação encontrando a direção da dependência entre os sinais. Esta abordagem tem a grande vantagem de dispensar estimativas da ordem do modelo, tempo morto e escolha da estrutura do modelo. O método é ilustrado através de sua aplicação a um exemplo de simulação e a dados obtidos de uma planta termoelétrica industrial, sendo validado a partir do conhecimento do processo.

\section{Introdução}

Muitas plantas industriais utilizam malhas de controle regulatório para possibilitar uma operação estável de seus processos e garantir que seus produtos satisfaçam parâmetros mínimos de qualidade exigidos por seus clientes. Neste contexto, um grande esforço é realizado pelas empresas para que suas plantas sejam operadas de forma a assegurar isto. Mas para que isso aconteça é essencial que o controle regulatório esteja funcionando apropriadamente. Levantamentos na indústria verificaram que cerca de $63 \%$ das malhas de controle apresentavam problemas que reduzem a eficiência do processo, entre as quais, $38 \%$ relacionadas a problema de sintonia de controladores, $19 \%$ relacionadas a estrutura de controle e apenas $6 \%$ relacionadas ao processo (Bialkowski, W. 1993). Estudos sobre o desempenho de malhas de controle apontam que as razões de baixo desempenho estão relacionados não somente a problemas de sintonia mas, também a problemas em equipamentos tais como válvulas com agarramento (N.F.Thornhill et al.,1997). Pesquisas comprovaram ainda que malhas de controle com comportamento oscilatório aumentam a variabilidade da qualidade do produto final, diminuem o tempo de vida útil do equipamento, reduzem produção, e até mesmo podem interromper a operação da planta (Karra et al., 2009). Ferramentas para avaliar o desempenho de controladores industriais através de uma combinação de resultados estatísticos como a detecção de oscilação pelo cálculo do erro acumulado (IAE) entre sucessivos cruzamentos por zeros (Hägglund, 1995), índice de desempenho baseado em variância mínima, entre outras estatísticas tradicionais, já é possível ser encontrado no mercado. O método utilizado neste trabalho é baseado na função de auto-correlação (ACF - Autocorrelation Function em inglês) e foi apresentado por Thornhill et al. (2003b), com proposta de melhorias feitas em (Kara et al., 2009), utilizando gráficos de densidade espectral de potência (Power Spectral Density - PSD em inglês) para identificar e separar as prováveis frequências de oscilação do sinal. Os sinais com frequência de oscilação semelhante e com energia maior que um certo limiar nesta frequência podem ser agrupados, para identificar qual deles é a fonte da oscilação. Os principais métodos para indicar a fonte, baseados em relações de causa e efeito, são a causalidade de Granger (Granger, 1969; Yuan et al., 2012) e a Transferência de Entropia (Schreiber, 2000). Um método baseado em identificação de sistemas foi proposto em (Marques et al., 2013). Neste artigo, um método baseado em identificação não pa- 
ramétrica é utilizado, com o grande diferencial de dispensar escolhas relacionadas a ordem e estrutura do modelo e estimativas do tempo morto.

Este artigo está organizado da seguinte forma: na Seção 2 é feita uma descrição do processo a ser analisado. A metodologia é proposta na Seção 3, sendo descrito o algoritmo de detecção de oscilações e proposto o algoritmo para identificar sua causa raiz. $\mathrm{Na}$ Seção 4 os algoritmos são aplicados a um exemplo da literatura e aos dados do processo industrial, com as conclusões sendo apresentadas na Seção 5.

\section{Descrição do Processo}

Uma central termoelétrica (CTE) tem como finalidade a geração de energia elétrica a partir da energia liberada em forma de calor através da queima de combustíveis. Neste processo, ilustrado de forma simplificada na Figura 1, são utilizados uma caldeira para geração de vapor d'água, seguida de uma turbina, gerador, condensador e aquecedores. A CTE utiliza como combustíveis os fluidos resultantes de processos siderúrgicos desencadeados dentro da própria usina, podendo ser BFG (Blast Furnace Gas - Gás de Alto Forno), COG (Coke Oven Gas - Gás de Coqueria), LDG (Lins Donawitz Gas - Gás de Aciaria) ou Alcatrão (TAR - Óleo resultante do processo de fabricação do coque). O vapor gerado na caldeira é armazenado no tubulão superior. Após ser superaquecido o vapor é conduzido à turbina. Esta tem como função converter a energia entálpica do vapor superaquecido em movimento mecânico que, por sua vez, é usado para acionar o gerador elétrico. $\mathrm{O}$ vapor de exaustão da turbina segue para o condensador onde passa ao estado líquido. O condensado acumulado na parte inferior do condensador é bombeado para os aquecedores de baixa pressão, segue para o desaerador e então é bombeado para os aquecedores de alta pressão seguindo para o economizador e retornando para o tubulão superior da caldeira fechando o ciclo. Os aquecedores de baixa pressão préaquecem o condensado através do vapor da $3^{\mathrm{a}}$ e $4^{\mathrm{a}}$ extração da turbina, enquanto que os aquecedores de alta pressão utilizam vapor da $1^{\mathrm{a}}$ e $2^{\mathrm{a}}$ extração da turbina. $\mathrm{O}$ pré-aquecimento do condensado eleva a temperatura da água de alimentação para temperaturas superiores a $200^{\circ} \mathrm{C}$ aumentando a eficiência energética e a estabilidade na caldeira. A função do desaerador é retirar ar do condensado e evitar corrosão. O sistema de geração de vapor conta ainda com dois aquecedores de vapor de baixa pressão ( $L P 1$ e $L P 2$ ), que recebem vapor da $2^{a}$ extração da caldeira e do by-pass da linha principal de vapor para turbina. A caldeira da CTE é do tipo aquatubular,ou seja, o vapor é produzido dentro de tubos enquanto que os gases de combustão circulam externos a eles. Resumidamente a fornalha consiste numa câmara com queimadores onde ar e combustíveis são misturados e queimados transferindo parte do calor para a água gerando vapor saturado que é enviado para dois superaquecedores. Eles estão localizados dentro da fornalha e recebem calor por radiação e convecção, eliminando por completo as gotículas de água produzindo vapor superaquecido. Um sistema de spray atemperador que garante o controle da temperatura do vapor que sai da caldeira através da injeção de água em alta pressão no vapor.

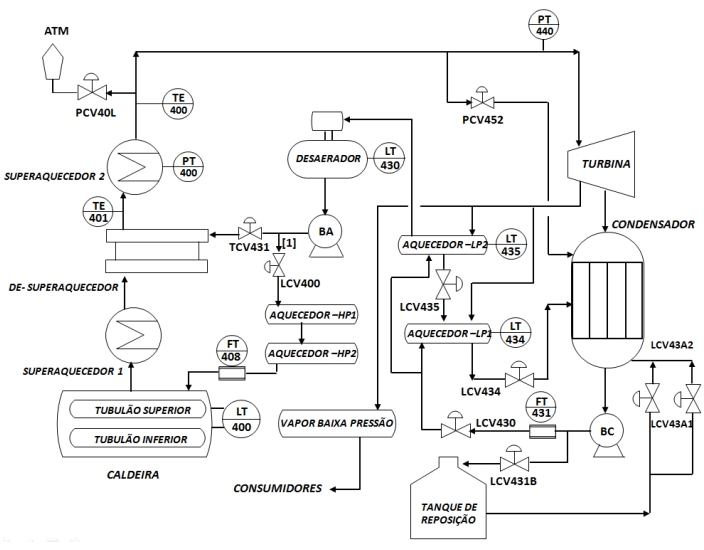

Figura 1. Fluxograma simplificado da CTE

\section{Detecção de oscilação e causa raiz}

\subsection{Caracterização do sinal oscilatório}

O PSD é obtido através da transformada de Fourier da autocorrelação do sinal e permite comparar o espectro de frequências de diferentes sinais. As frequências dominantes são aquelas que possuem maior energia, que pode ser medida integrando na frequência as potências da faixa de frequências considerada. A intensidade da oscilação pode ser quantificada usando a potência (Thornhill et al.,2003). A caracterização do sinal oscilatório é feita através do índice de regularidade do período $(r)$. O desvio padrão é verificado para vários períodos calculados, resultando no parâmetro $r: r>1$ indica regularidade no período do sinal oscilatório. Outro índice aqui utilizado é o proposto por (Miao et al.,1999), baseado no decaimento da ACF.

Em (Karra et al.,2009), o método baseado na ACF é melhorado. O PSD do sinal é plotado, a faixa de frequências com energia significativa é identificada, e um filtro baseado na inversa da Transformada de Fourier é utilizado para obter o correspondente sinal no tempo. Caso o período deste sinal seja regular, conclui-se que esta frequência está presente no sinal. O método permite tratar o caso de múltiplas frequências de forma bastante natural. Uma vez detectadas as frequências de oscilação nos sinais, o próximo passo é indicar o sinal que o originou, pois é comum um sinal oscilatório se propagar pela planta afetando os demais. A forma mais simples de fazer esta análise é indicar como provável fonte os sinais 
com maior energia, medida na faixa do espectro correspondente à oscilação para cada sinal. A causalidade de Granger tem sido utilizada para esta finalidade, usando para isto modelos ARMA ajustados aos dados em análise (Yuan et al., 2012). Como a inclusão de todas as variáveis gera modelos de baixa qualidade, um método baseado em identificação de sistemas foi proposto em (Marques et al., 2013).

Na próxima subseção um método de identificação não paramétrica é utilizado, com a vantagem de dispensar estimativas de tempo morto, da ordem do modelo e de estruturas de modelo a serem utilizadas.

\subsection{Direção da interação entre sinais via identifica- ção não paramétrica.}

O método proposto a seguir não permite detectar a existência de conexões entre as variáveis, mas apenas sua direção, requerendo um teste preliminar para verificar se as variáveis estão de alguma forma conectadas. Um teste clássico é o cálculo da correlação no tempo ou em frequência, substituído aqui pela verificação dos sinais com frequência de oscilação comum. Em (Marques et al., 2013), a validação de um modelo paramétrico entre dois sinais permitiu concluir sobre a direção desta interação. Propomos aqui o uso do método da Estimação da Função de Transferência Empírica (ETFE - Empirical Transfer Function Estimate em inglês), calculada como a razão entre Transformada de Fourier do sinal de saída e a Transformada de Fourier do sinal de entrada. Um fator de suavização (smoothing - smo), pode ser empregado para suavizar a previsão espectral usando a janela de Hamming. O resultado da estimação pode ser visualizado através do Gráfico de Bode da Função de Transferência (FT) estimada. É sabido que sistemas de fase não mínima apresentam diagramas de Bode no qual o atraso de fase depende do número de polos e zeros. Sistemas causais (mais polos que zeros) tem atraso de fase quando a frequência varia de zero a infinito, enquanto para sistemas não causais há um avanço de fase. Como os sinais analisados no contexto dos problemas deste artigo são originados de malhas de controle estáveis, com predomínio de dinâmicas de primeira ordem, esta abordagem revelase bastante adequada. A presença de tempo morto produz atrasos puros de fase. A conclusão sobre a direção da interação entre os sinais vem da análise da fase dos modelos não paramétricos, com o atraso de fase indicando a direção correta da interação. O módulo do Gráfico de Bode (GB) é um bom indicador da força da interação entre as variáveis, sendo utilizado aqui o valor do ganho para a frequência mais baixa calculada, que corresponde ao ganho estático da FT. Embora o uso do método ETFE seja bem conhecido para estimação não paramétrica, os autores desconhecem seu uso para indicação da direção da interação entre os sinais.

\section{Metodologia}

A CTE apresentada neste trabalho possui ao todo 58 malhas de controle abrangendo um número significativo de variáveis, entre as quais citamos pressão, vazão, nível, temperatura e variáveis analíticas como teor de oxigênio entre outras. Dentre as 58 malhas existentes foram selecionadas as 39 malhas mais importantes. Para isso foram levados em consideração fatores tais como criticidade da malha para o processo, estabilidade operacional da planta e sob o ponto de vista econômico, malhas com potencial de redução de perdas de energia por combustão incompleta dos gases.

Os dados para análise foram coletados utilizando-se o software de auditoria de malhas de controle TriCLPM (TriSolutions Control Loop Performance Monitor). O TriCLPM é uma ferramenta para auditoria de desempenho de controladores de processo que reduz o tempo gasto com análise do controle ao apontar as malhas com maior potencial de melhoria e ajudar a diagnosticar as causas de desempenho insatisfatório dos controles regulatórios. Para o diagnóstico de oscilações, o software permite gerar gráficos do espectro das malhas que podem ser comparadas, procurando frequências comuns. $\mathrm{O}$ algoritmo aqui discutido permite fazer isto de forma automática, reduzindo o tempo para a busca de oscilações comuns nas malhas.

A estimação não paramétrica é feita usando a função etfe do Matlab, considerando um sistema com uma saída e múltiplas entradas, com o objetivo de verificar as entradas para as quais ocorre atraso de fase. A tendência dos sinais é removida, não sendo feita normalização. Desta forma, modelos não paramétricos são obtidos um a um para todas as variáveis em análise consideradas com saída, tendo as demais variáveis consideradas como entradas. A análise para todas as variáveis é resumida em um gráfico para cada saída, indicando por uma barra o módulo do $\mathrm{GB}$, com valor negativo para atraso é positivo para avanço para cada variável considerada como entrada (ver Fig.5).

\section{Aplicações}

\subsection{Exemplo de simulação.}

O primeiro exemplo desta Seção é utilizado para ilustrar a aplicação da metodologia da Seção 3.2, identificando a direção da interação entre as variáveis para um modelo conhecido obtido de (Ding et al., 2008), para o qual a casualidade de Granger foi analisada, sendo dado por,

$$
x(n, 1)=0.8 x(n-1,1)-0.5 x(n-2,1)+0.4 z(n-1,1)+r 1(n, 1)
$$

$y(n, 1)=0.9 y(n-1,1)-0.8 y(n-2,1)+r 2(n, 1)$ 
$z(n, 1)=0.5 z(n-1,1)-0.2 z(n-2,1)+0.5 y(n-1,1)+r 3(n, 1)$

Onde $r_{1}, r_{2}, r_{3}$ são ruídos brancos com distribuição gaussiana com média zero e variância $\sigma^{2}$ de 0.3 , 1.0 e 0.2 respectivamente. Neste modelo, $y$ afeta $z$ e $x$, e $z$ afeta $x$. O fator de suavização (smo) utilizado neste exemplo foi 8. Na Figura 2 é mostrado o GB tendo $y$ como saída e as variáveis $x$ e $z$ como entradas: como há um avanço de fase para as duas variáveis, conclui-se que estas variáveis não afetam $y$, ou dito de outra forma, a direção da interação não é de $z$ e $x$ para $y$. Na Figura 3 é mostrado o GB tendo a variável $z$ como saída e as variáveis $x$ e $y$ como entradas. A fase é atrasada para $y$ como entrada e avançada para $x$ como entrada. Finalmente, na Figura 4 é mostrado o GB tendo $x$ como saída e $y$ e $z$ como entradas, e neste caso a fase está atrasada para as duas variáveis de entrada. Logo, ambas variáveis afetam $x$ e o módulo de $z$ para $x(-4 \mathrm{~dB})$ é maior que de y para $\mathrm{x}(-11.5 \mathrm{~dB})$, uma vez que $z$ afeta diretamente $x$ de acordo com (2). Na Figura 5 são resumidos os resultados para as 3 variáveis, sendo mostrado para cada variável o valor do ganho da FT para baixas frequências (altura da barra) e se a fase avança (gráfico de barra acima de zero) ou atrasa (gráfico de barra abaixo de zero). O valor do módulo da FT dá uma indicação da força da relação existente entre as variáveis. As entradas 1,2,3 correspondem às variáveis $y, z, x$ respectivamente. Para uma dada variável de saída, basta verificar para quais variáveis as barras correspondentes estão abaixo de zero, o que indica que elas afetam a variável de saída (atraso de fase). Na Figura 5 observa-se que $y$ afeta $x$ e $z$, que $z$ afeta $x$ e que $x$ não afeta nenhuma variável. Caso estas três variáveis contivessem uma mesma frequência de oscilação, a fonte seria indubitavelmente a variável $y$.

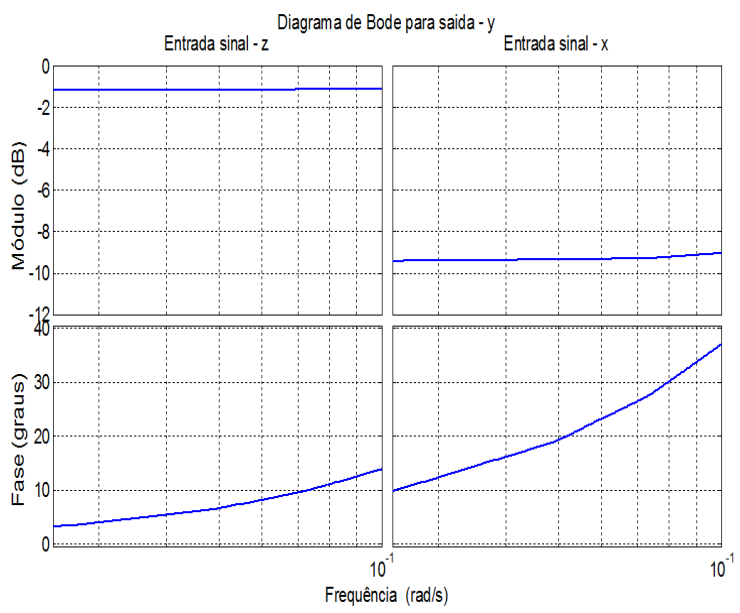

Figura 2. Gráfico de Bode - entrada ' $z x$ ' saída ' $y$ '

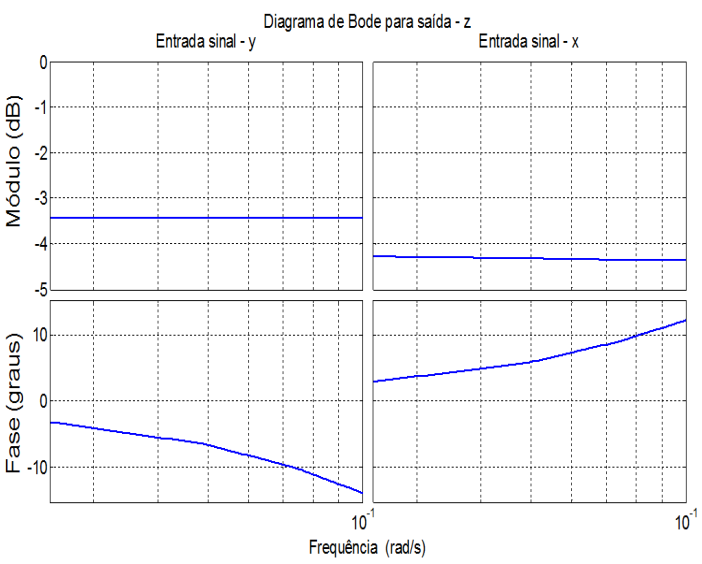

Figura 3. Gráfico de Bode - entrada ' $y x$ ' saída ' $z$ '

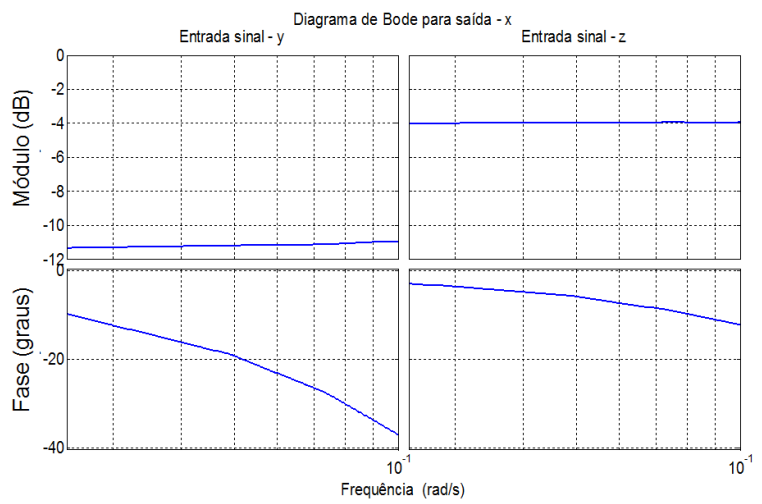

Figura 4. Gráfico de Bode - entrada ' $y z$ ' saída ' $x$ '
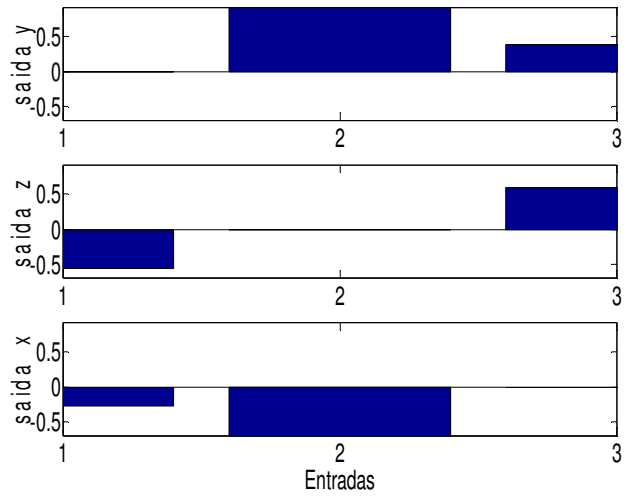

Figura 5. Direção da interação entre as variáveis

\subsection{Exemplo com dados da indústria}

Neste exemplo, os algoritmos para detecção de oscilações são aplicados para identificar as malhas com mesma frequência de oscilação, para a seguir identificar a malha que é fonte da oscilação utilizando o método proposto na Seção 3.2.

Foram utilizados dados coletados do processo por um período de 12 horas a partir das 6:00 horas da manhã, pois é no período diurno que as malhas sofrem constantes distúrbios em função das manobras 
operacionais realizadas para adequar a demanda de vapor e energia elétrica produzida em função da disponibilidade dos gases combustíveis. Durante esse período de 12 horas os dados foram coletados com uma taxa de amostragem de $5 \mathrm{~s}$, totalizando assim 43200 pontos para cada variável. O tempo de amostragem foi escolhido pela equipe que configurou o software TriCLPM, de modo a representar adequadamente as variáveis analisadas.

O método de detecção de oscilações foi aplicado as 39 malhas, e destas 6 apresentaram comportamento oscilatório mais significativo na frequência 0.0102 $\mathrm{rad} / \mathrm{s}$ (período de 10.26 minutos). O procedimento usual nestes casos é priorizar a detecção e mitigação do efeito de oscilações que mais impactam o processo. O PSD destes 6 sinais é mostrado na Figura 7. O algoritmo utilizado seleciona apenas as variáveis que tem energia maior que um certo limiar, neste caso de $30 \%$ da máxima energia encontrada. O passo seguinte é identificar a fonte desta oscilação. Uma análise baseada no conhecimento sobre o processo foi realizada, para validar o algoritmo proposto, usando a Figura 1 como referência.

A variação da pressão da caldeira PIC400 provoca uma variação no nível do tubulão LIC400. O tubulão da caldeira funciona como um separador entre a água no estado de vapor saturado e a água líquida saturada com bolhas de vapor. Quando a pressão cai, o volume das bolhas misturadas à água líquida cresce significativamente, fazendo com que o nível do tubulão suba. Entretanto, como a queda de pressão normalmente é devido a um aumento da vazão de vapor saindo do tubulão e como a temperatura água de alimentação (reposição) é mais fria que a temperatura da água na caldeira, com o passar do tempo, a tendência é que após essa subida inicial do nível, o mesmo tenda a cair depois de algum tempo. $\mathrm{O}$ efeito contrário também ocorre quando há um aumento da pressão do vapor; inicialmente o nível cai, mas depois de algum tempo volta a subir. Ao variar a pressão da caldeira PIC400 imediatamente ocorre a variação do nível de água do tubulão. O controle do nível é realizado em cascata com o controle de vazão de água de alimentação para o tubulão FIC408 através da válvula LCV400. Esta atuação afeta o nível do desaerador LIC430, que é controlado pela válvula LCV430, posicionada na saída do condensador. A variação da pressão de vapor contribui também para a variação do vapor de baixa pressão extraído da turbina para os aquecedores de baixa pressão LP1 e LP2 cujo nível é controlado pela LCV434 e LCV435 respectivamente, influenciando o nível LIC434.

As variações no controle de vazão FIC408 através da válvula LCV400 contribui para a queda de pressão no ramo [1] de saída de água para o spray provocando assim variações no controle de temperatura do desuperaquecedor TIC400 que é realizado através da válvula TCV431.

Ao controlar a temperatura do vapor TIC400, a válvula TCV431 altera a pressão de água no recalque da bomba de condensado BC. Ao variar a pressão na bomba ocorre em pequena escala a variação na vazão de condensado do desaerador e consequentemente no controle do nível LIC430. Estas relações estão representadas na Figura 6.

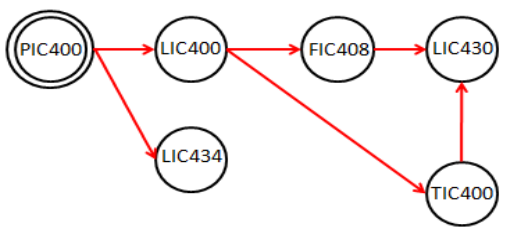

Figura 6. Grafo de interação entre as 6 malhas via análise do processo
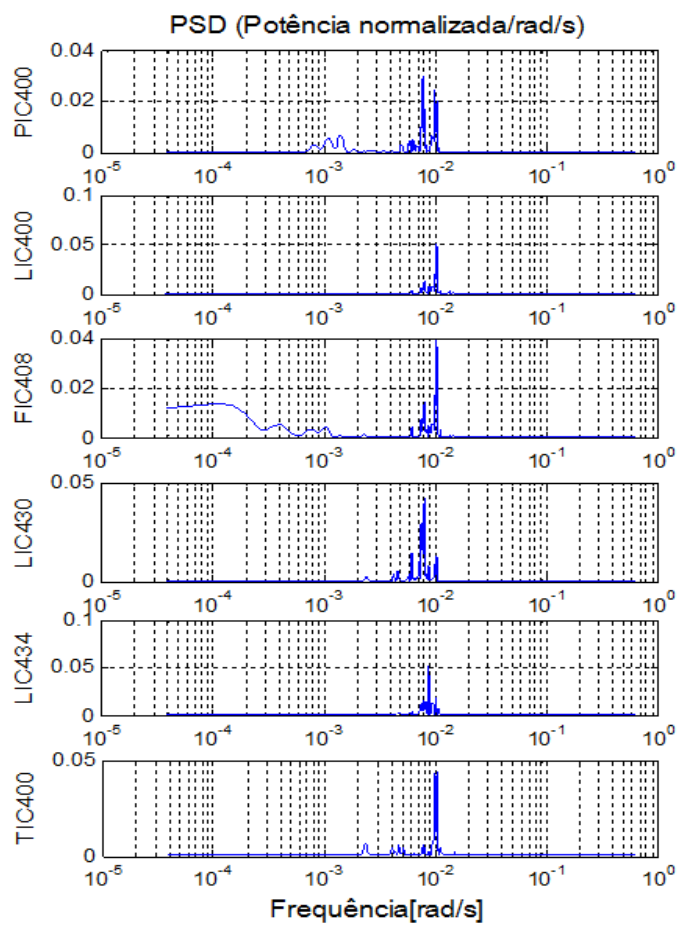

Figura 7. PSD dos 6 sinais com frequência de oscilação semelhante da CTE

O método proposto na Seção 3.2 foi então aplicado obtendo os modelos não paramétricos para cada uma das 6 variáveis consideradas como saída e as demais 5 variáveis como entradas. Os dados extraídos dos gráficos de Bode para as 6 variáveis são mostrados na Figura 8.

As entradas 1,2,3,4,5 e 6 correspondem às malhas PIC400, LIC434, FIC408, LIC400, LIC430 e TIC400 respectivamente. Como o maior módulo obtido para os modelos foi 3.58, analisaremos apenas relações entre variáveis cujo módulo seja maior que $10 \%$ deste valor. Um método para definir um limiar deve ser posteriormente proposto, o que é comum em todos os métodos que investigam direção de interação. A partir da análise das Figuras $8 \mathrm{a}$ ) até $8 \mathrm{f}$ ), podese construir o grafo da Fig.9. Por exemplo, da Fig. 8b) conclui-se que LIC434 é afetada apenas por PIC400 e que FIC408 é afetada por LIC400 e TIC400 e afeta LIC430. Observa-se a similaridade entre os grafos das Figuras 6 e 9, e que TIC400 e 
PIC400 afetam outras variáveis, mas não são por ela afetadas, ficando claro que é nelas que deve ser concentrar a busca pelas causas da oscilação. O efeito de LIC400 em TIC400 é desprezado na Fig. 9, pois apesar de existir, seu efeito é pequeno como mostra a amplitude da barra correspondente usando o limiar (Fig 8f).

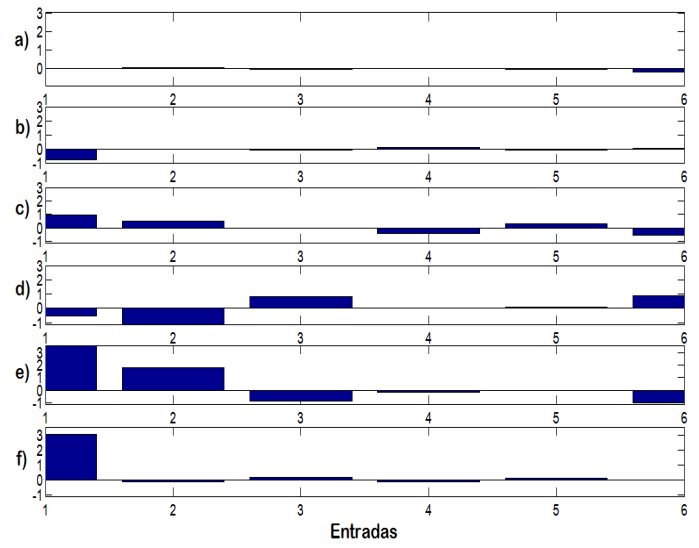

Figura 8. Direção da interação entre as variáveis

A não detecção do efeito de LIC400 sobre TIC400 é um exemplo de que embora fisicamente esta relação exista, seu efeito não é perceptível a partir dos dados, dada a fraca relação existente, podendo ser desconsiderada. Os dados mostram uma relação entre LIC400 e LIC430, mas esta relação pode ocorrer através de FIC408. A proposta feita em (Marques et al., 2013) permite elucidar se este efeito é direto ou indireto, e não feita aqui devido à limitação de espaço, por não alterar as conclusões sobre a causa raiz. A alteração da temperatura TIC400 produz variações de vazão através da válvula TCV431 (Ver Fig.1), que por sua vez pode afetar a vazão FT408. Esta análise não foi feita no processo mas foi indicada pelos dados. Percebe-se assim que os dados fornecem informações que não são quantificadas na análise do processo, capturando de forma mais fidedigna as relações existentes entre as variáveis na situação de operação dada. Esta análise valida o método proposto, e poderia ter sido usada sem a necessidade de levantar informações detalhadas sobre o processo para indicar as malhas PIC400 e TIC400 como aquelas a serem investigadas como causa raiz.

\section{Conclusão}

Este artigo apresenta uma proposta de metodologia para a indicação da causa raiz de oscilações para dados de processos industriais. As oscilações são detectadas utilizando-se um método da literatura baseado na função de autocorrelação. Um método de identificação não paramétrica é então proposto para indicar a direção da relação entre as variáveis com frequência de oscilação comum. A proposta é aplicada a um exemplo de simulação e a dados de uma central termoelétrica. No primeiro exemplo a validação se deu pelo conhecimento do modelo utilizado.

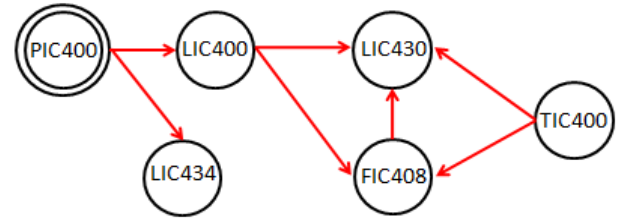

Figura 9. Interação entre as 6 malhas método proposto

No segundo exemplo, um grafo da relação entre as variáveis baseado no conhecimento do processo foi comparado ao grafo obtido pela metodologia proposta. Ficou evidenciada sua similaridade e para fins de determinação da causa raiz chega-se à mesma conclusão. A metodologia proposto pode ser estendida para outras áreas de aplicação. Sinais não oscilatórios também podem ter a direção de suas interrelações determinadas, substituindo o método de detecção de oscilações por algum método que indique os sinais entre os quais exista correlação, avaliada no tempo ou na frequência.

\section{Referências Bibliográficas}

Aguirre, L. A. (2007). Introdução à Identificação de Sistemas Editora UFMG, 3 a edição.

Bialkowski,W. (1993). A view from both sides of a gap. In Dreams versus reality, number 94(11) in 1, pages 19-27. Pulp and Paper, Canada.

Ding, M., Chen,Y., Bressler, S.L, Granger causality: Basic theory and application to neuroscience, Elsevier Science, 2008.

Granger, C. W. J, Investigating causal relations by econometric models and cross-spectral methods, Econometrica, vol. 37, no. 3, pp. 424-438, August 1969. DOI: 10.2307/1912791

Hägglund, T. (1995). A control-loop performance monitor. Control Engineering Practice, 3(11):1543 - 1551. DOI: 10.1016/0967-0661(95)00164-P

Horch, A., Thornhill, N., Seborg, D., Laing, D., Michael Grimble, P. M., Xial, H., Boll, M., Nohr, M., Corsi, S., Bannauer, M., and Zimmer, G. (2007). Benchmarking control loop with oscillation and stiction. In Process control performance assessment, Advances in industrial controls, pp.227-257. Springer London, London. 10.1007/978-1-84628-624-7 7

Karra, S., Karim, M. N. (2009). Comprehensive methodology for detection and diagnosis of oscillatory control loops. Control Engineering Practice, 17(8):939-956. DOI: 10.1016/j.conengprac.2009.02.015

Marques, V. M., Munaro, C. J., Shah, L. S. Data-based Causality Detection from a System Identification Perspective. European Control Conference, pp.2453-2458, Zurich-CH, julho/2013.

Schreiber, T. Measuring information transfer, Phys. Rev. Lett., vol. 85 , no. 2 , pp. 461-464, 2000. DOI: 10.1103/PhysRevLett.85.461

Thornhill, N. F., Hägglund, T. Detection and Diagnosis of Oscillations in Control, ControlEng. Practice, Vol. 5, No.10, pp. 1343-1354, 1997. DOI: 10.1016/S0967-0661(97)00131-7

Thornhill, N. F., Huang, B., Zhang, H. (2003). Detection of multiple oscillations in control loops. Journal of Process Control, 13(1):91 - 100. DOI: 10.1016/S0959-1524(02)00007-0

Yuan, T., Qin, S. J. Root cause diagnosis of plant-wide oscillations using granger causality, 8th IFAC Symposium on Advanced Control of Chemical Processes, Singapore 2012. 